\title{
Properties of the Free-Standing Two-Dimensional Copper Monolayer
}

\author{
Li-Ming Yang,, Thomas Frauenheim, ${ }^{2}$ and Eric Ganz ${ }^{3}$ \\ ${ }^{1}$ School of Chemistry and Chemical Engineering, Huazhong University of Science and Technology, Wuhan 430074, China \\ ${ }^{2}$ Bremen Center for Computational Materials Science, University of Bremen, Am Falturm 1, 28359 Bremen, Germany \\ ${ }^{3}$ Department of Physics, University of Minnesota, 116 Church St., SE, Minneapolis, MN 55416, USA
}

Correspondence should be addressed to Li-Ming Yang; lmyang.uio@gmail.com

Received 8 March 2016; Accepted 8 September 2016

Academic Editor: Giuseppe Compagnini

Copyright (C) 2016 Li-Ming Yang et al. This is an open access article distributed under the Creative Commons Attribution License, which permits unrestricted use, distribution, and reproduction in any medium, provided the original work is properly cited.

\begin{abstract}
We use density functional theory to study a free-standing $2 \mathrm{D}$ copper monolayer. We find that the Cu monolayer is stable in $15 \mathrm{ps}$ $a b$ initio molecular dynamics simulations up to $1200 \mathrm{~K}$. Due to the smaller number of bonds per atom in the 2D layer compared to the $3 \mathrm{D}$ bulk, we observe a significantly enhanced energy per bond ( 0.92 versus $0.58 \mathrm{eV} / \mathrm{bond})$. This is similar to the increase in bond strength going from $3 \mathrm{D}$ diamond to $2 \mathrm{D}$ graphene. We predict various properties of this material, including band structure and density of states. The free-standing 2D Cu monolayer is hexagonal close packed and is the global minimum structure. One valence electron from each atom is delocalized and is donated into a $2 \mathrm{D}$ nearly free electron gas.
\end{abstract}

\section{Introduction}

Ultrathin materials including graphene, translation metal dichalcogenides, and boron nitride have generated great interest and exhibit fascinating properties including high carrier mobility [1], quantum Hall effect [2], extraordinary thermal conduction [3], magnetic resonance, and superconductivity [4]. Generally, these ultrathin materials have been based on single layers of lamellar van der Waals materials. These materials are stable in few- or even single-layer structures, due to the strong intralayer chemical bonding and weak van der Waals interlayer interaction [5-8]. In contrast to van der Waals materials, metal atoms have a strong preference for three-dimensional (3D) close packed structures. Therefore, monolayer free-standing metallic structures with numerous unsaturated atoms are difficult to stabilize and their synthesis has been challenging. In this paper, we will discuss the stability and properties of free-standing monolayer $\mathrm{Cu}$ films.

Bulk copper is estimated to have been discovered in 9000 $\mathrm{BC}$ in the Middle East [9]. Copper is a ductile and malleable metal with very high thermal and electrical conductivity. Copper catalysts have numerous applications, including oxidation of alcohols, NO, and CO [10]. Copper oxides include high temperature superconductors with scientific and technological importance [11]. Copper is a key cofactor in many biological oxidation-reduction reactions [12, 13]. Copper complexes at the active site in metalloproteins and important contributions to the biological functions $[14,15]$. Cyclic $\mathrm{Cu}_{n}$ structural motifs (especially $\mathrm{Cu}_{3}$ ) have been reported to be building blocks of various organic and inorganic systems [16-18]. Copper is essential to all living organisms as a trace dietary mineral because it is a key constituent of the respiratory enzyme complex cytochrome $\mathrm{c}$ oxidase. $\mathrm{Cu}(111)$ is widely used as substrate to fabricate numerous $2 \mathrm{D}$ sheets including graphene [19] and boron nitride [20].

Dimensionality is crucial for material properties. The same chemical compounds can exhibit dramatically different properties depending on whether they are arranged in $0 \mathrm{D}$, $1 \mathrm{D}, 2 \mathrm{D}$, or $3 \mathrm{D}$ crystal structures. This is demonstrated by different carbon materials which can vary from $0 \mathrm{D}$ clusters and fullerenes [21] to 1D nanotubes [22], 2D graphene [23], and 3D graphite and diamond. We see that structures of different dimensionality have unique properties.

Copper clusters have also demonstrated size-dependent properties that are completely different from the bulk phase [24]. This demonstrates the importance of quantum confinement and the size or shape of the object on its properties, 
functionalities, and applications. There have also been interesting experiments on the adsorption of small molecules (such as azobenzene and molecular switches) on the $\mathrm{Cu}(111)$ surface [25].

In 2014, Zhao et al. reported the experimental fabrication of small free-standing atomically thick iron patches of up to ten atoms in graphene pores [26]. One can imagine making such patches out of other metals as well. For example, this method could inspire the experimental fabrication of small free-standing $\mathrm{Cu}$ monolayers. Motivated by these experimental results on free-standing Fe films, as well as by recent computational predictions of free-standing 2D Au and Ag monolayers $[27,28]$, we decided to investigate the freestanding $\mathrm{Cu}$ monolayer.

In this paper, we present $a b$ initio studies of the freestanding $\mathrm{Cu}$ monolayer. We demonstrate that this material is quite stable, as it can be annealed for $15 \mathrm{ps}$ up to $1200 \mathrm{~K}$. Molecular dynamics movies are included in the Supplemental Information in Supplementary Material available online at http://dx.doi.org/10.1155/2016/8429510, and we also evaluate the bond length distribution. We present calculations of the band structure and density of states and compare the 2D material to bulk 3D Cu. We will also compare our results to calculations of free-standing $2 \mathrm{D}$ gold and silver monolayers $[27,28]$. We hope that these results will inform experimental efforts in this direction. We expect that these materials will exhibit new and unusual properties.

\section{Computational Methods}

We calculated the electronic structure and total energy using density functional theory (DFT) with the plane-wave pseudopotential (PWPP) technique as implemented in the Vienna $a b$ initio Simulation Package (VASP) [29]. Ionelectron interactions were implemented with the projectoraugmented wave (PAW) [30,31] method. The generalized gradient approximation (GGA) and the PBE functional [32] were used with a $500 \mathrm{eV}$ limit for the plane-waves. An energy threshold of $10^{-6} \mathrm{eV}$ and a force threshold of $10^{-2} \mathrm{eV} / \AA$ were used. The $2 \mathrm{D}$ atom sheet was located in the $x y$ plane. To avoid the influence of neighboring layers in the periodic simulation, a $20 \AA$ spacing in the $z$ direction was used. For geometry optimization and electronic structure calculations, the Brillouin zone was sampled with a $24 \times 24 \times 1 \Gamma$-centered Monkhorst-Pack (MP) [33] K-point grid.

Phonon calculations were performed using the finite displacement method [34] using CASTEP in Materials Studio 7.0 [35]. The local-density approximation with ultrasoft pseudopotentials was used. The SCF tolerance was $5 \times$ $10^{-7} \mathrm{eV} /$ atom, and the force tolerance was $10^{-3} \mathrm{eV} / \AA$, and the energy cutoff was $330 \mathrm{eV}$, at the ultrafine setting. The Brillouin zone was sampled with a $24 \times 24 \times 1 \mathrm{MP}$ grid for both phonon dispersion and phonon density of states. A $7 \times 7$ supercell was used with an $8.0 \AA$ cutoff radius. For the Brillouin zone, an average distance of $0.003 \AA^{-1}$ between Monkhorst-Pack mesh $q$-points was used.

$A b$ initio Born-Oppenheimer molecular dynamics simulations were performed to assess the thermal stability of the $\mathrm{Cu}$ monolayer. The scalar-relativistic DFT-D and the
Tkatchenko-Scheffler (TS) method were used in CASTEP [35] in Materials Studio 7.0. The NVT ensemble was used. Typical simulations ran for up to $10 \mathrm{ps}$ with a time step of 1.0 fs (parameters: accuracy fine, $\mathrm{SCF}=3 \times 10^{-6}$, smearing $=$ 0.04, DIIS = 20, Nosé-Hoover method [36], Nosé $Q=2$, Nosé chain length $=2$, and fixed center of mass). Materials Studio was also used to create the initial structures and visualize the results. We use periodic boundary conditions for all calculations

Crystal structure predictions were performed using the evolutionary algorithm as implemented in the USPEX code [37]. In these calculations, initial structures are randomly produced using planar group symmetry. All the newly produced structures are relaxed, and the relaxed energies are used to select structures as parents for the next generation. Each subsequent generation is produced by carefully designed variation operators such as heredity and soft mutation. We considered systems with up to 18 atoms in the unit cell and used 30 structures in each generation, with $60 \%$ of the lowest-enthalpy structures allowed to produce the next generation through heredity $(60 \%)$, lattice mutation $(30 \%)$, and atomic permutation (10\%). In addition, two lowestenthalpy structures were allowed to survive into the next generation. The structure relaxations during the evolutionary algorithm were performed using the PBE functional as implemented in VASP. The Visualization for Electronic and Structural Analysis software (VESTA, series 3) [38] was used for visualization and plotting.

\section{Results and Discussion}

We use the USPEX search program to find the global minimum and low energy isomers of a $2 \mathrm{D} \mathrm{Cu}$ monolayer [37]. The hexagonal close packed ground state structure of the free-standing $\mathrm{Cu}$ monolayer is shown in Figure 1. Other low-lying isomers shown in Figure 1 include trigonal + tetragonal, square, and tetracoordinate configurations. These isomers have relative energies of $0.15,0.30$, and $0.71 \mathrm{eV} /$ atom, respectively. We also observed that the open honeycomb lattice of the $\mathrm{Cu}$ monolayer transformed to the square lattice during geometry optimization. This close packed structure maximizes the number of bonds in the plane and is the expected structure. These in-plane bonds act to stabilize and maintain the planar configuration of the sheet. The model has $P 6 / \mathrm{mmm}$ (\#191) space group symmetry. The unit cell consists of a single $\mathrm{Cu}$ atom with lattice constant $a=b=2.44 \AA$ and $\mathrm{Cu}-\mathrm{Cu}$ bond length of $2.44 \AA$. We note that this freestanding $2 \mathrm{D} \mathrm{Cu}$ monolayer holds great potential to be realized experimentally over a small hole.

The electron charge density is shown in Figure 2(a) and can be used to evaluate the chemical bonding between copper atoms of the monolayer. Further insight into the bonding interaction comes from the analysis of the electron localization function (ELF) shown in Figure 2(b). This provides a good description of electron delocalization in molecules [39] and solids [40] and is a useful tool for chemical bond classification [41]. The ELF values are very low. The distribution of electrons in the $2 \mathrm{D} \mathrm{Cu}$ monolayer is similar to that for the $3 \mathrm{D} \mathrm{Cu}(111)$ surface as shown in Figure 2. 


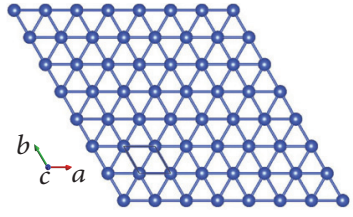

(a)

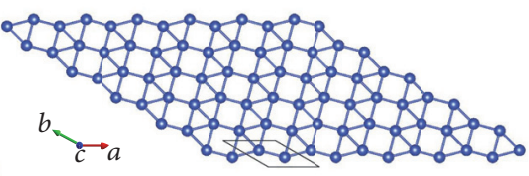

(b)

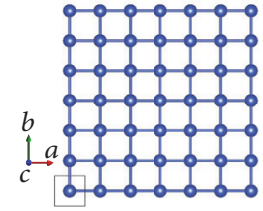

(c)

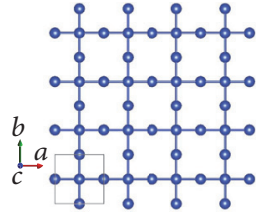

(d)

FIGURE 1: Different motifs of Cu monolayer: (a) hexagonal close packed, (b) trigonal + tetragonal, (c) square, and (d) tetracoordinate.

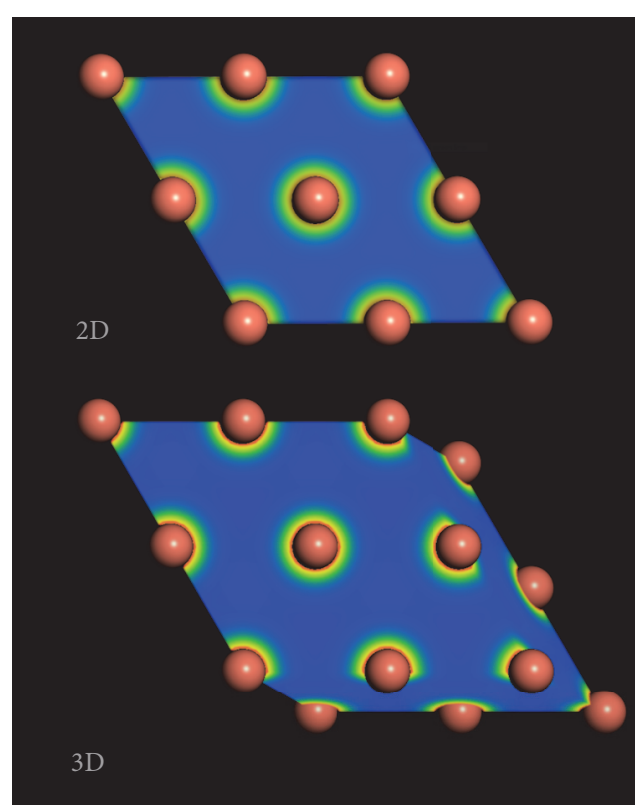

(a)

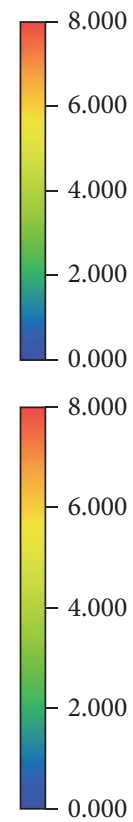

0.000

Figure 2: (a) Charge density and (b) ELF of the 2D Cu monolayer and 3D bulk Cu.

Next, we evaluated the cohesive energy for the $2 \mathrm{D}$ freestanding monolayer and the bulk 3D material.

$$
\begin{aligned}
& E_{\text {coh } 2 \mathrm{D}}=\frac{\left(x E_{\text {Cu-atom }}-E_{\text {Cu-monolayer }}\right)}{x}, \\
& E_{\text {coh3D }}=\frac{\left(x E_{\text {Cu-atom }}-E_{\text {Cu-bulk }}\right)}{x} .
\end{aligned}
$$

$E_{\mathrm{Cu} \text {-atom }}, E_{\mathrm{Cu} \text {-monolayer }}$, and $E_{\mathrm{Cu} \text {-bulk }}$ are the total energies of a single $\mathrm{Cu}$ atom, one unit cell of the $2 \mathrm{D} \mathrm{Cu}$ monolayer, and one unit cell of 3D bulk $\mathrm{Cu}$, respectively. Based on our results, the $2 \mathrm{D} \mathrm{Cu}$ monolayer and the $3 \mathrm{D}$ bulk have cohesive energies of 2.76 and $3.48 \mathrm{eV} /$ atom, respectively. For comparison, using the same computational method, the cohesive energies of a $2 \mathrm{D}$ and bulk $3 \mathrm{D}$ Au layer are 2.82 and $3.11 \mathrm{eV}$ per atom, respectively [28]. The bond strength is increased in comparison to the bulk due to the smaller number of bonds per atom ( 6 versus 12). Therefore each bond is stronger in the sheet than in the bulk $(0.92 \mathrm{eV} /$ bond versus $0.58 \mathrm{eV} /$ bond, resp.).

We tested the stability of this system by calculating the phonon dispersion, which is shown in Figure 3 [34]. All the

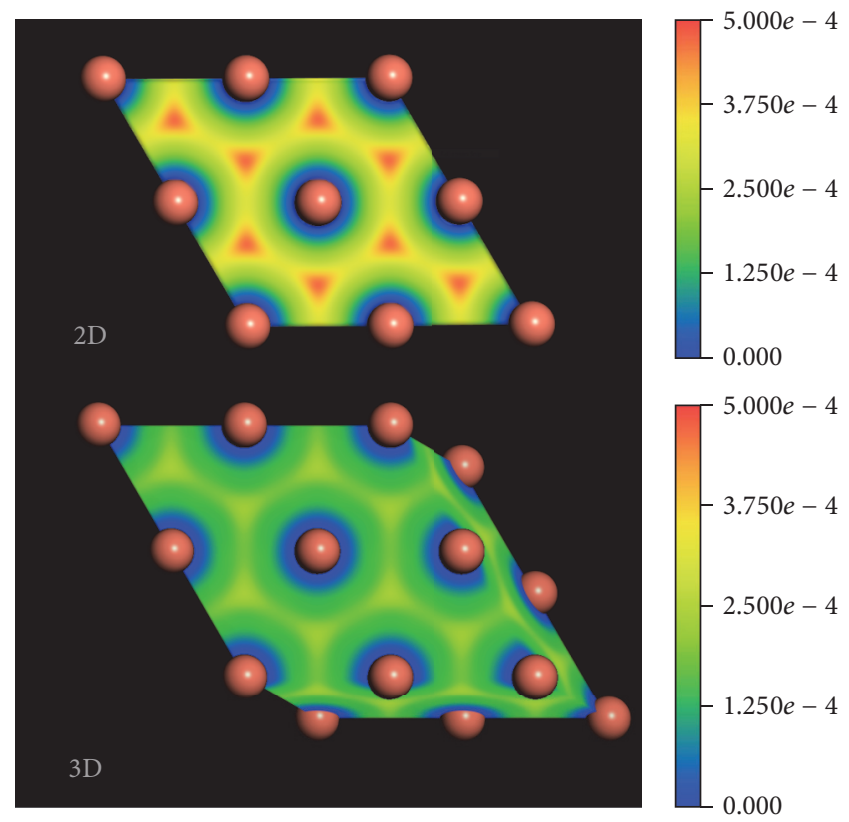

(b)

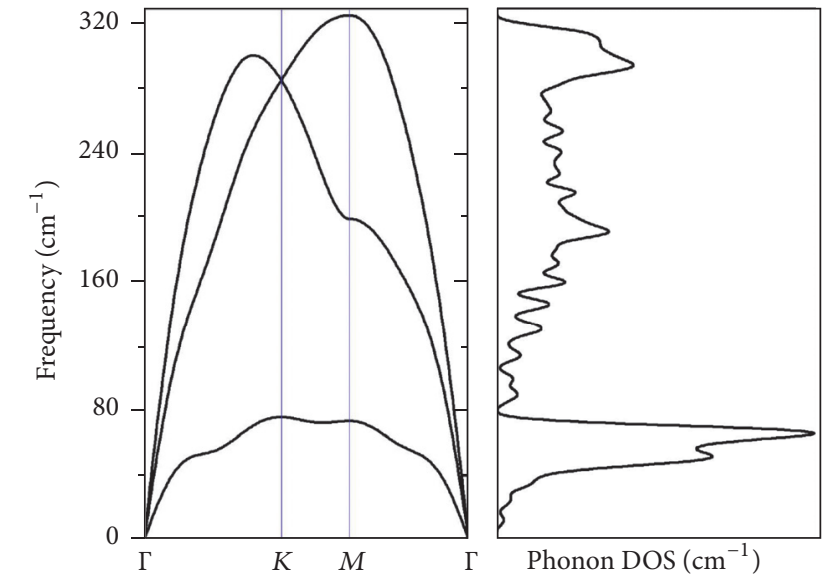

FIGURE 3: Phonon dispersion and phonon density of states of the 2D $\mathrm{Cu}$ monolayer. $\Gamma(0,0,0), M(0,1 / 2,0)$, and $K(1 / 3,2 / 3,0)$ refer to special points in the first Brillouin zone in reciprocal space.

frequencies are real, indicating kinetic stability. The largest observed frequency is $325 \mathrm{~cm}^{-1}$. 


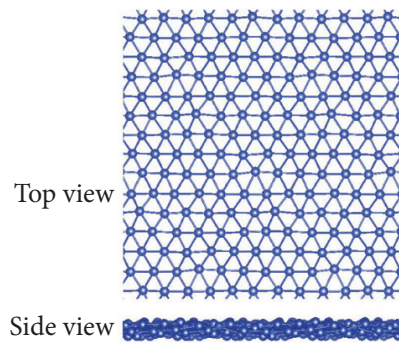

$500 \mathrm{~K}$

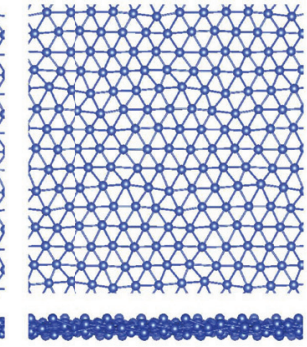

$800 \mathrm{~K}$

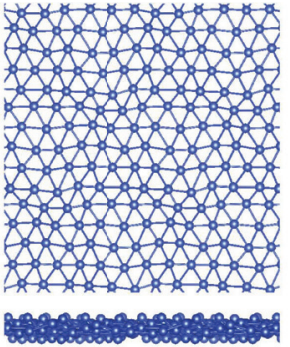

$1200 \mathrm{~K}$

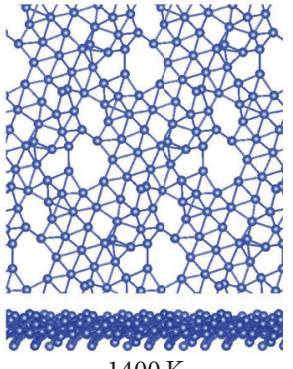

$1400 \mathrm{~K}$

Figure 4: Snapshots of the final frame of each molecular dynamics simulation from 500 to $1400 \mathrm{~K}$ (top and side views). Bonds to atoms outside this section exist but are not shown.

To verify the thermal stability of this new material at both ambient conditions and also at elevated temperatures, we have performed $a b$ initio Born-Oppenheimer molecular dynamics simulations. Periodic $5 \times 6$ and $8 \times 8$ supercells were used in the MD simulations. A series of individual 4$15 \mathrm{ps}$ simulations were carried out at temperatures of 500 , 800,1200 , and $1400 \mathrm{~K}$. Snapshots taken at the end of each of these simulations are shown in Figure 4. A survey of bond length extensions shows an increase of up to $16 \%, 29 \%$, and $33 \%$ during last $1 \mathrm{ps}$ of the 500,800 , and $1200 \mathrm{~K}$ runs. These results demonstrate that the $\mathrm{Cu}$ monolayer has good thermal stability and can maintain its structural integrity during brief $15 \mathrm{ps}$ annealing up to $1200 \mathrm{~K}$. At $1400 \mathrm{~K}$, the system is starting to melt (holes appearing and diffusion) already on these short time scales. These numbers can be compared to the melting point of $1358 \mathrm{~K}$ for bulk $\mathrm{Cu}$. At elevated temperatures, we may consider that this is a quasi-2D monolayer. The liquid regime for this material will be explored in a separate paper. A movie of one of the molecular dynamics simulations at $800 \mathrm{~K}$ for $0.5 \mathrm{ps}$ is available in the Supplemental Information.

In Figure 5, we show the results of the bond length distribution for the copper monolayer at temperatures of $500 \mathrm{~K}, 800 \mathrm{~K}$, and $1200 \mathrm{~K}$. We observe a significant range of bond lengths, primarily due to motion in the $Z$ direction. Bond length extensions up to $3.3 \AA$ are observed.

To get insight into the electronic properties of this system, we have computed the band structure and the density of states (DOS). As shown in Figure 6(a), the 2D Cu monolayer shows a band structure typical for metals. The metallic character of the $\mathrm{Cu}$ monolayer is demonstrated by the Fermi level $(E=$ 0 ) being located inside the bands and no observation of a bandgap at energy $E=0$. Therefore, the $\mathrm{Cu}$ monolayer is a nonmagnetic metal. The partial density of states (PDOS) is also plotted in Figure 6(a) to visualize the contributions of individual orbitals. There is a sharp peak under the Fermi level mainly contributed by the $\mathrm{Cu}-d$ states. The states at the Fermi level have major contributions from $\mathrm{Cu}-p$ and $\mathrm{Cu}-d$ states, with $p>d>s$ (right). There is apparent hybridization between the $\mathrm{Cu}-p$ and $\mathrm{Cu}-d$ states. The $\mathrm{Cu}-d$ states are close to and below the Fermi level. From the band structure in Figure 6(a), we see that one band crosses the Fermi level. This band is highly dispersive and crosses the Fermi level halfway up (between the $\Gamma$ point and the $K$-point). This band corresponds to a similar free electron band in bulk 3D Cu

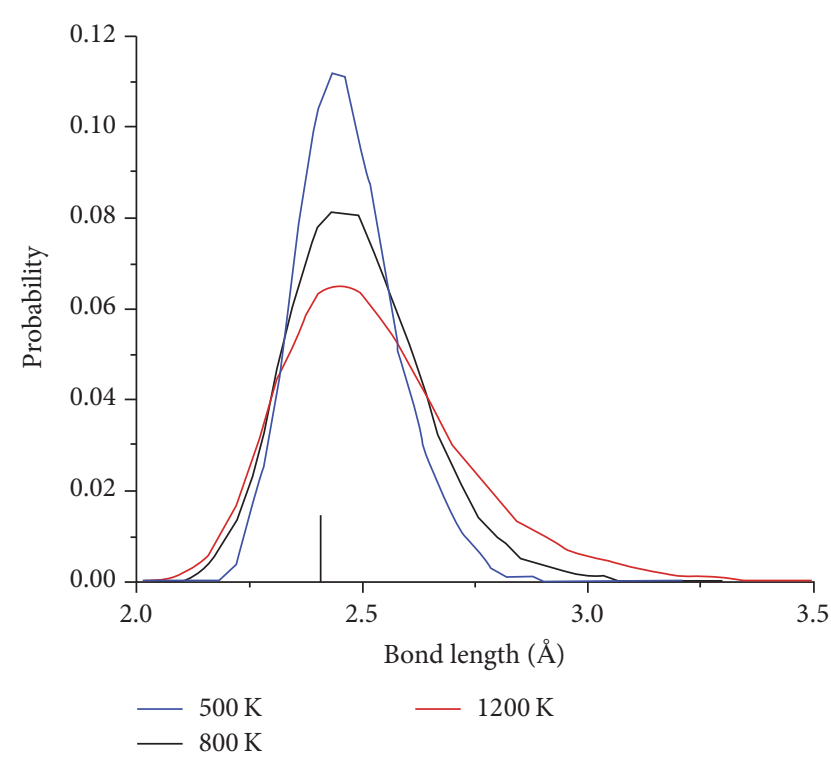

FIGURE 5: Bond length distribution for the copper monolayer at $500 \mathrm{~K}, 800 \mathrm{~K}$, and $1200 \mathrm{~K}$. A black vertical line is drawn at $2.41 \AA$ to show the $0 \mathrm{~K}$ bond length. We observe that there is a wide distribution of bond lengths due primarily to motion in the $Z$ direction.

and indicates a donation of one electron to the free electron gas. We can see that one valence electron from each atom is delocalized and participates in a nearly free homogeneous electron gas. In Figure 6(b), we show the band structure of bulk 3D $\mathrm{Cu}$ for comparison. We see that the $d$ band DOS are somewhat different in the two cases, although both are metallic.

In Table 1, we compare the predicted properties of the free-standing $\mathrm{Cu}, \mathrm{Ag}$, and Au monolayers [27, 28]. All of these free-standing 2D monolayers have been studied using the same parameters and computational methods. At the lowest temperature of $500 \mathrm{~K}$, we observe that the copper and gold systems show very low bond length extensions. Cu has the highest bulk cohesive energy space (by 10\%) and the highest bulk melting point (by $1 \%$ ). Therefore, it is quite surprising that, as a 2D layer, copper ends up somewhat weaker than gold. We see that $\mathrm{Au} 2 \mathrm{D}$ free-standing monolayer has the highest cohesive energy (by 2\%). Furthermore, we see that 
TABLE 1: Comparison of the predicted properties of $\mathrm{Cu}, \mathrm{Ag}$, and $\mathrm{Au}$ free-standing 2D monolayers.

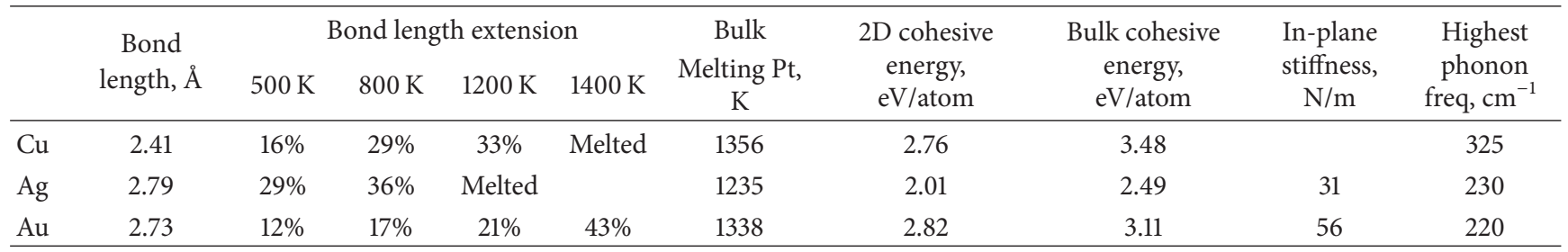
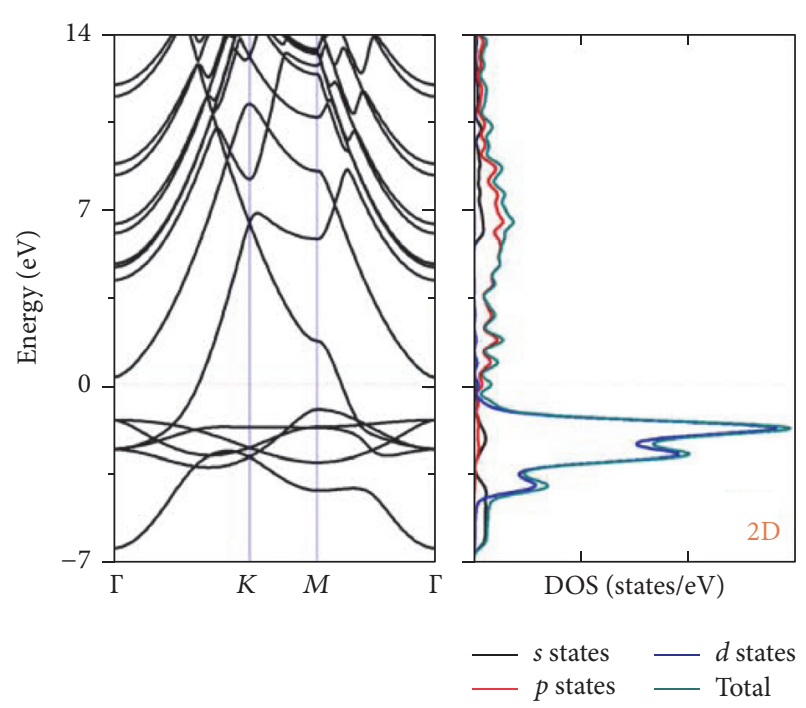

(a)

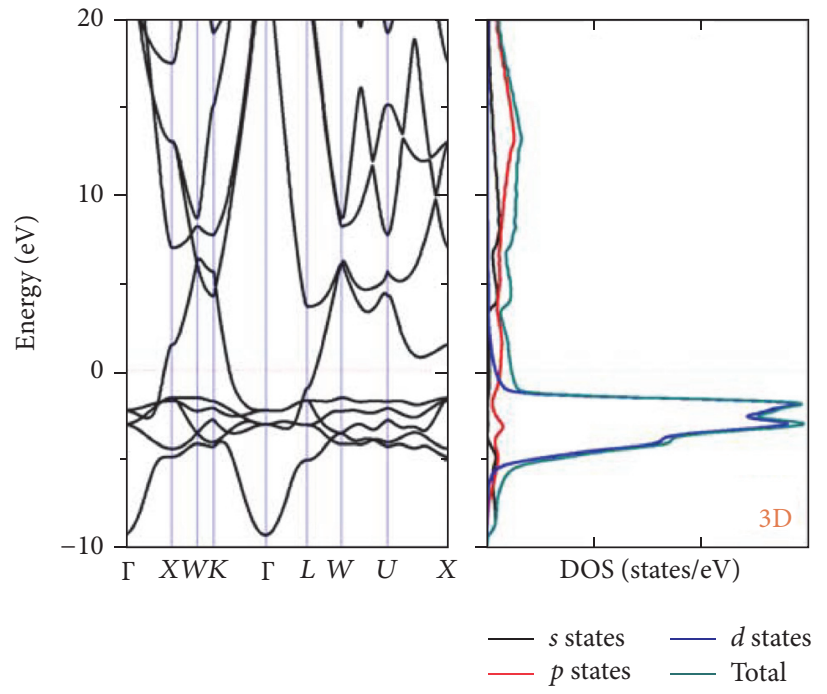

(b)

Figure 6: (a) Band structure and DOS of the free-standing 2D Cu monolayer. (b) Band structure and DOS of the bulk 3D Cu. The Fermi level is at $0 \mathrm{eV}$.

the $2 \mathrm{D}$ Au monolayer can be annealed for 10 ps up to $1400 \mathrm{~K}$ without destruction of the framework in that time, while the $\mathrm{Cu}$ layer only went up to $1200 \mathrm{~K}$. We see that silver has the lowest bulk cohesive energy, which corresponds to the lowest $2 \mathrm{D}$ cohesive energy and the lowest observed 2D annealing temperature ranges. We conclude that the free-standing 2D $\mathrm{Au}$ layer is unusually robust, but the $\mathrm{Cu}$ layer should also be experimentally accessible.

\section{Conclusions}

In summary, we have studied the free-standing $2 \mathrm{D} \mathrm{Cu}$ monolayer. We have used molecular dynamics simulations to demonstrate that the bonding network survives $15 \mathrm{ps}$ annealing runs up to $1200 \mathrm{~K}$. This material turns out to be slightly less strong than the free-standing 2D gold layer, but more resilient than the free-standing Ag monolayer. The 2D $\mathrm{Cu}$ monolayer is HCP with planar hexacoordinate bonding. With only six atoms per bond, the individual bond energy is substantially larger than the corresponding bulk value. The material is metallic with one electron donated into the free electron gas. We used an evolutionary algorithm to search the $2 \mathrm{D}$ space to confirm that this is a global minimum structure. The phonon spectrum was tested to ensure stability against negative modes. A wide range of bond lengths are observed.
We hope that researchers will be inspired to study this system or other similar free-standing 2D metal monolayers.

\section{Competing Interests}

The authors declare that they have no competing interests.

\section{Acknowledgments}

Li-Ming Yang gratefully acknowledges the support from startup fund (2006013118 and 3004013105) and independent innovation research fund (0118013090) from Huazhong University of Science and Technology and National Natural Science Foundation of China (Grant no. 21673087). LiMing Yang and Eric Ganz thank Matthew Dornfeld for his assistance. The authors thank the Minnesota Supercomputer Institute for support.

\section{References}

[1] F. Miao, S. Wijeratne, Y. Zhang, U. C. Coskun, W. Bao, and C. N. Lau, "Phase-coherent transport in graphene quantum billiards," Science, vol. 317, no. 5844, pp. 1530-1533, 2007.

[2] Y. Zhang, Y.-W. Tan, H. L. Stormer, and P. Kim, "Experimental observation of the quantum Hall effect and Berry's phase in graphene," Nature, vol. 438, no. 7065, pp. 201-204, 2005. 
[3] A. A. Balandin, "Thermal properties of graphene and nanostructured carbon materials," Nature Materials, vol. 10, pp. 569581, 2011.

[4] H. He, P. Bourges, Y. Sidis et al., "Magnetic resonant mode in the single-layer high-temperature superconductor $\mathrm{Tl}_{2} \mathrm{Ba}_{2} \mathrm{CuO}_{6+\delta}$," Science, vol. 295, no. 5557, pp. 1045-1047, 2002.

[5] M. Chhowalla, H. S. Shin, G. Eda, L.-J. Li, K. P. Loh, and H. Zhang, "The chemistry of two-dimensional layered transition metal dichalcogenide nanosheets," Nature Chemistry, vol. 5, no. 4, pp. 263-275, 2013.

[6] C. Ataca, H. Şahin, and S. Ciraci, "Stable, single-layer $\mathrm{MX}_{2}$ transition-metal oxides and dichalcogenides in a honeycomblike structure," Journal of Physical Chemistry C, vol. 116, no. 16, pp. 8983-8999, 2012.

[7] S. Lebègue, T. Björkman, M. Klintenberg, R. M. Nieminen, and O. Eriksson, "Two-dimensional materials from data filtering and ab initio calculations," Physical Review X, vol. 3, no. 3, Article ID 031002, 7 pages, 2013.

[8] M. Pandey, A. Vojvodic, K. S. Thygesen, and K. W. Jacobsen, "Two-dimensional metal dichalcogenides and oxides for hydrogen evolution: a computational screening approach," The Journal of Physical Chemistry Letters, vol. 6, no. 9, pp. 1577-1585, 2015.

[9] CSA-Discovery Guides, A Brief History of Copper, http://csa .com.

[10] M. Shelef, "Selective catalytic reduction of $\mathrm{NO}_{x}$ with $\mathrm{N}$-free reductants," Chemical Reviews, vol. 95, no. 1, pp. 209-225, 1995.

[11] V. E. Henrich and P. A. Cox, The Surface Science of Metal Oxides, Cambridge University Press, New York, NY, USA, 1994.

[12] W. Kaim and B. Schwederski, Bioanorgansiche Chemie, Teubner, Stuttgart, Germany, 1991.

[13] F. A. Cotton, G. Wilkinson, C. A. Murillo, and M. Bochmann, Advanced Inorganic Chemistry, John Wiley \& Sons, New York, NY, USA, 6th edition, 1999.

[14] E. I. Solomon, U. M. Sundaram, and T. E. Machonkin, "Multicopper oxidases and oxygenases," Chemical Reviews, vol. 96, no. 7, pp. 2563-2605, 1996.

[15] R. H. Holm, P. Kennepohl, and E. I. Solomon, "Structural and functional aspects of metal sites in biology," Chemical Reviews, vol. 96, no. 7, pp. 2239-2314, 1996.

[16] H. V. R. Dias, H. V. K. Diyabalanage, M. A. RawashdehOmary, M. A. Franzman, and M. A. Omary, "Bright phosphorescence of a trinuclear copper(I) complex: luminescence thermochromism, solvatochromism, and 'concentration luminochromism," Journal of the American Chemical Society, vol. 125, no. 40, pp. 12072-12073, 2003.

[17] X.-S. Wang, H. Zhao, Z.-R. Qu et al., "An unprecedented 8-fold interpenetrating diamondoid-like coordination polymer containing a $\mathrm{Cu}_{4}^{+}\left(\mathrm{RCO}_{2}\right)_{4}$ cluster as connecting node," Inorganic Chemistry, vol. 42, no. 19, pp. 5786-5788, 2003.

[18] H. Eriksson and M. Håkansson, "Mesitylcopper: tetrameric and pentameric," Organometallics, vol. 16, no. 20, pp. 4243-4244, 1997.

[19] X. Li, W. Cai, J. An et al., "Large-area synthesis of high-quality and uniform graphene films on copper foils," Science, vol. 324, no. 5932, pp. 1312-1314, 2009.

[20] S. Joshi, D. Ecija, R. Koitz et al., "Boron nitride on $\mathrm{Cu}(111)$ : an electronically corrugated monolayer," Nano Letters, vol. 12, no. 11, pp. 5821-5828, 2012.

[21] H. W. Kroto, J. R. Heath, S. C. O’Brien, R. F. Curl, and R. E. Smalley, " $\mathrm{C}_{60}$ : Buckminsterfullerene," Nature, vol. 318 , no. 6042, pp. 162-163, 1985.
[22] S. Iijima, "Helical microtubules of graphitic carbon," Nature, vol. 354, no. 6348, pp. 56-58, 1991.

[23] K. S. Novoselov, A. K. Geim, S. V. Morozov et al., "Electric field in atomically thin carbon films," Science, vol. 306, no. 5696, pp. 666-669, 2004.

[24] K. J. Taylor, C. L. Pettiette-Hall, O. Cheshnovsky, and R. E. Smalley, "Ultraviolet photoelectron spectra of coinage metal clusters," The Journal of Chemical Physics, vol. 96, no. 4, pp. 33193329, 1992.

[25] E. R. McNellis, J. Meyer, and K. Reuter, "Azobenzene at coinage metal surfaces: role of dispersive van der Waals interactions," Physical Review B, vol. 80, no. 20, Article ID 205414, 2054.

[26] J. Zhao, Q. Deng, A. Bachmatiuk et al., "Free-standing singleatom-thick iron membranes suspended in graphene pores," Science, vol. 343, no. 6176, pp. 1228-1232, 2014.

[27] L.-M. Yang, T. Frauenheim, and E. Ganz, “The new dimension of silver," Physical Chemistry Chemical Physics, vol. 17, no. 30, pp. 19695-19699, 2015.

[28] L.-M. Yang, M. Dornfeld, T. Frauenheim, and E. Ganz, "Glitter in a 2D monolayer," Physical Chemistry Chemical Physics, vol. 17, no. 39, pp. 26036-26042, 2015.

[29] G. Kresse and J. Hafner, "Ab initio molecular dynamics for liquid metals," Physical Review B, vol. 47, no. 1, pp. 558-561, 1993.

[30] P. E. Blöchl, "Projector augmented-wave method," Physical Review B, vol. 50, no. 24, pp. 17953-17979, 1994.

[31] G. Kresse and D. Joubert, "From ultrasoft pseudopotentials to the projector augmented-wave method," Physical Review B, vol. 59, no. 3, pp. 1758-1775, 1999.

[32] J. P. Perdew, K. Burke, and M. Ernzerhof, "Generalized gradient approximation made simple," Physical Review Letters, vol. 77, no. 18 , pp. 3865-3868, 1996.

[33] H. J. Monkhorst and J. D. Pack, "Special points for Brillouinzone integrations," Physical Review B, vol. 13, no. 12, pp. 5188$5192,1976$.

[34] G. Kresse, J. Furthmüller, and J. Hafner, “Ab initio force constant approach to phonon dispersion relations of diamond and graphite," Europhysics Letters, vol. 32, no. 9, p. 729, 1995.

[35] S. J. Clark, M. D. Segall, C. J. Pickard et al., "First principles methods using CASTEP," Zeitschrift fur Kristallographie, vol. 220, no. 5-6, pp. 567-570, 2005.

[36] G. J. Martyna, M. L. Klein, and M. Tuckerman, "Nosé-Hoover chains: the canonical ensemble via continuous dynamics," The Journal of Chemical Physics, vol. 97, no. 4, pp. 2635-2643, 1992.

[37] A. R. Oganov and C. W. Glass, "Crystal structure prediction using ab initio evolutionary techniques: principles and applications," The Journal of Chemical Physics, vol. 124, no. 24, Article ID 244704, 2006.

[38] K. Momma and F. Izumi, "VESTA 3 for three-dimensional visualization of crystal, volumetric and morphology data," Journal of Applied Crystallography, vol. 44, no. 6, pp. 1272-1276, 2011.

[39] A. D. Becke and K. E. Edgecombe, "A simple measure of electron localization in atomic and molecular systems," The Journal of Chemical Physics, vol. 92, no. 9, pp. 5397-5403, 1990.

[40] A. Savin, O. Jepsen, J. Flad, O. K. Andersen, H. Preuss, and H. G. von Schnering, "Electron localization in solid-state structures of the elements: the diamond structure," Angewandte Chemie (International Edition in English), vol. 31, no. 2, pp. 187-188, 1992.

[41] B. Silvi and A. Savin, "Classification of chemical bonds based on topological analysis of electron localization functions," Nature, vol. 371, no. 6499, pp. 683-686, 1994. 

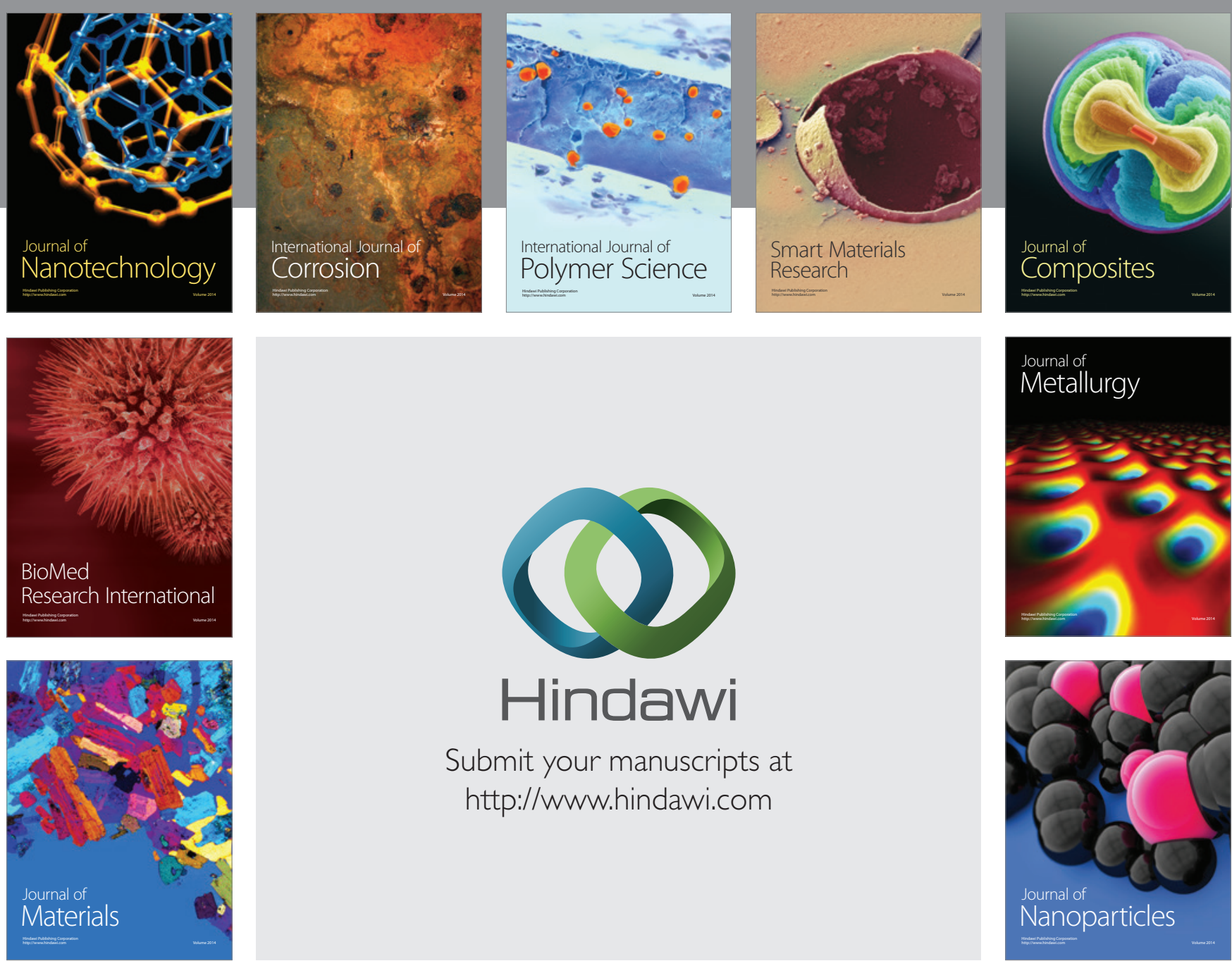

\section{Hindawi}

Submit your manuscripts at

http://www.hindawi.com

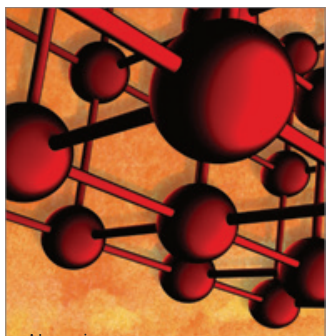

Materials Science and Engineering
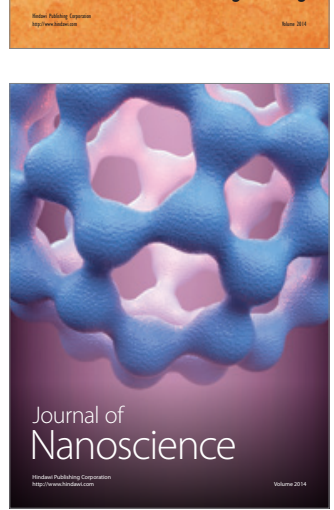
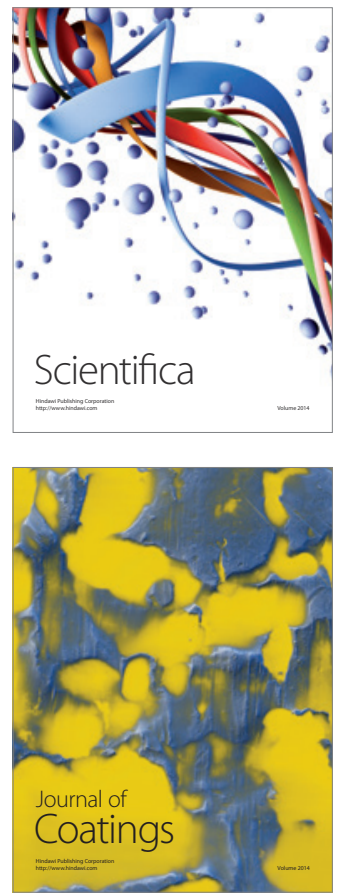
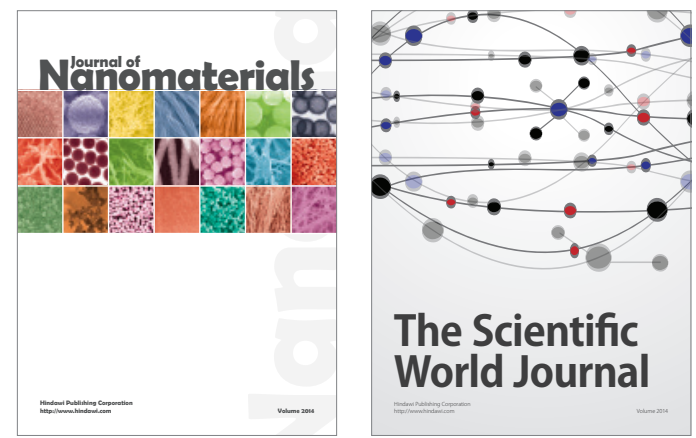

The Scientific World Journal
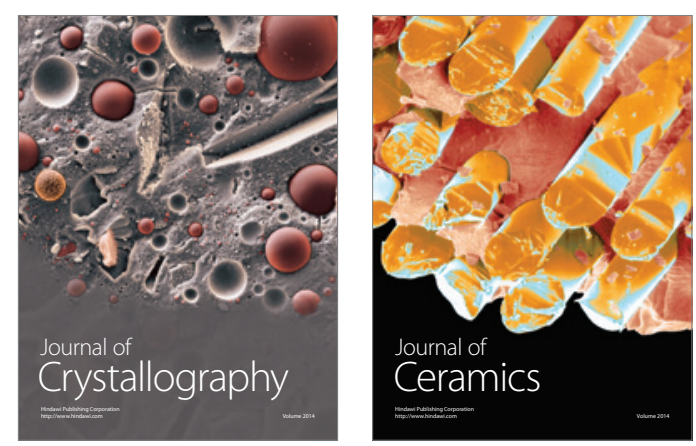
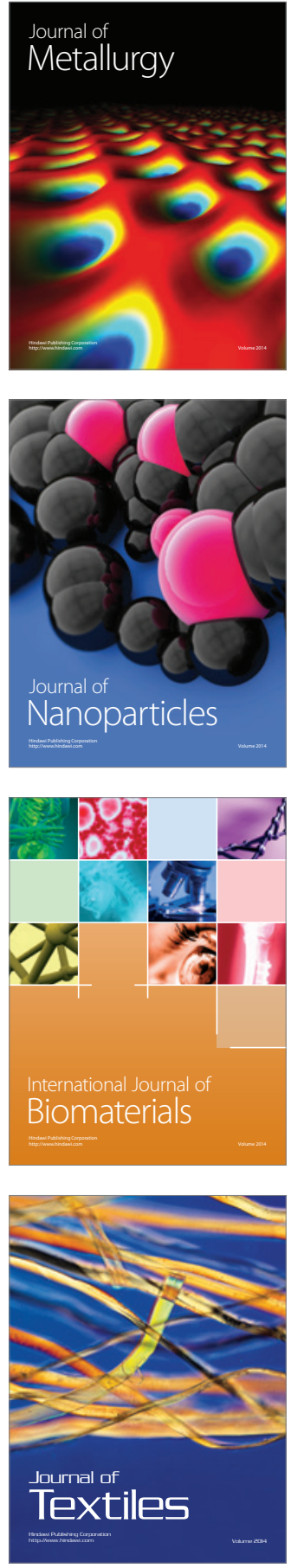